\title{
SUFFICIENT CLASSES OF STRATEGIES IN DISCRETE DYNAMIC PROGRAMMING I: DECOMPOSITION OF RANDOMIZED STRATEGIES AND EMBEDDED MODELS*
}

\author{
E. A. FAINBERG
}

(Translated by Merle Ellis)

1. Introduction. One of the major questions that occurs in investigating problems of dynamic programming on an infinite time interval is: in which natural classes of strategies do there exist strategies that produce a pay-off uniformly close to the pure value? It is known that in the case of finite state and control sets, optimal stationary strategies exist [1] (this also follows from [2]). However, if the set of states or controls is infinite, then optimal (and even $\varepsilon$-optimal) stationary strategies need not exist [3], [4, Chap. 6, § 6, Example 2].

In the case of a countable state space $X$, a very general result was announced in [5, Theorem 2.1] that gives a description of such classes. Let $\Gamma$ denote the set of all mappings $F: X \rightarrow 2^{X}$ such that $x \in F(x)$ for all $x \in X$. A nonrandomized strategy is called an F-strategy if in any current state $x$ the control is chosen depending only on this state and the total time passed in $F(x)$ prior to the current instant of time. Markovian and tracking [6] strategies are special cases of $F$-strategies. Let $X^{*}$ be a state set where the pure value is different from 0 , or the pure value is equal to zero but controls exist on which the value of the optimality operator applied to the pure value is attained. According to Theorem 2.1 formulated in [5], for any $F \in \Gamma$ there exists an $F$-strategy that for all initial prehistories produces a pay-off uniformly close to the pure value (persistently $\varepsilon$ l-optimal strategy; a precise definition of persistently $\varepsilon l$-optimal strategies is given in $\S 6$ ) and is stationary on $X^{*}$. This theorem, on the one hand, elaborates the results of Everett [7] and Chitashvili [8], [9] on the sufficiency of strategies that are stationary on subsets in case $X$ is finite; on the other hand, it extends in various ways results on the sufficiency of stationary strategies [3], [10]-[12] and the sufficiency of Markov strategies [13]-[16], and gives a positive answer to a problem raised in [17] regarding the sufficiency of tracking strategies.

This article uses one of the two schemes suggested in [5] to prove Theorem 2.1 of [5]. We prove a stronger result (Theorem 6.2) that generalizes Theorem 2.1 in [5] in the following three directions: (1) a broader state set than $X^{*}$ is given where one can confine oneself to stationary controls; (2) wherever one cannot confine oneself to stationary controls, one can choose nonstationary strategies not only among the $F$-strategies but also from other natural classes of strategies; (3) the existence of persistently $\varepsilon l$-optimal strategies is proved for functions $l$ in a broader class than in Theorem 2.1 of [5].

We point out that the question of the sufficiency of stationary strategies on $X^{*}$ in the case of a countable chain was raised by $R$. Ya. Chitashvili in a lecture at the Third Colloquium-Seminar on the theory of random processes (Panaga, May 1982).

Note that the consideration of persistently $\varepsilon l$-optimal strategies for variable $l$ instead of $l \equiv 1$ allows one to take into account both the specifics of the negative models [13] (where one can set $l \equiv 1$ ) and the specifics of the positive models (where one cannot set $l=1$ [3] but can set $l$ equal to the pure value of the model [10]). Moreover,

* Received by the editor February 22, 1984. 
in the main results of this article (Theorems 6.1 and 6.2) $l(x)=0$ if optimal strategies exist for the initial state $x \in X$. Hence the results of the paper yield the existence of strategies that are optimal simultaneously for all prehistories terminating in states for which optimal strategies exist, if these states are viewed as initial states.

In proving the sufficiency of strategies that are stationary on some $Y \subseteq X$, the necessity of going over from the original model to a model with state set $Y$ arises. Here each time in the new model (which is said to be $Y$-embedded) corresponds to the time of the next hit in $Y$ in the original model, while the controls in the new model correspond to the intervals of the control trajectories between the times of hits in $Y$ in the original model. In $\S 5$ we propose a corresponding construction that in particular makes it possible in a general situation to apply theorems on the existence of stationary persistently $\varepsilon l$-optimal strategies in models with everywhere positive [12] or everywhere negative pure value (Theorem 9.1). We show that the pure values in the original and embedded models coincide on $Y$ (Theorem 5.1A) and that the question of the sufficiency of stationary strategies on $Y$ in the original model reduces to the question of the sufficiency of stationary strategies in the embedded model (Theorem 7.1).

To set up a correspondence between the original and the embedded model we introduce the notion of a $Y$-semi-embedded strategy. For such a strategy the choice of a control after the time of first hit in $Y$ is made depending on (i) the current state; (ii) the number of hits in $Y$ prior to the current instant of time, (iii) the time elapsed since the last hit in $Y$; (iv) the state at the time of the last hit in $Y$. We give two subsets of the set of all $Y$-semi-embedded strategies for which the control choice depends only on the quantities (i)-(iii) (the class of Y-embedded strategies) or depends only on (i), (iii) and (iv) (the class of $Y$-renewal strategies). Observe that $X$-embedded strategies are Markovian, while $X$-renewal strategies are stationary. In $\S 4$ we prove a theorem on the sufficiency of randomized $Y$-embedded strategies that extends the analogous familiar result for Markov strategies [18]. It is shown that the pure value of the class of $Y$-renewal strategies in the original model coincides on $Y$ with the pure value of the class of stationary strategies in the $Y$-embedded model (Theorem 5.1B).

To prove the sufficiency of nonrandomized strategies, we consider in $\S 3$ a theorem dealing with decomposition of randomized strategies (to study embedded models, such a theorem is necessary with respect to $Y$-semi-embedded or $Y$-embedded strategies). For arbitrary strategies, a decomposition theorem was proved by Krylov [1] in the case of countable state and control sets (it was indicated in [1] that an analogous assertion had been stated by I. V. Girsanov) and by Gikhman and Skorokhod [19] in the case of Borel state and control spaces. For Borel state and control spaces, a similar theorem was proved in [20] for Markov and semi-Markov strategies. In $\S \S 2$ and 3 of this paper, a sufficient condition is proved for the case of a countable state set and arbitrary control spaces (Condition 2.1) which a class of strategies must satisfy in order that the decomposition theorem (Theorem 3.1) hold for it. This condition is satisfied by many natural classes of strategies including the Markovian, $Y$-embedded, $Y$-semiembedded, tracking strategies and $F$-strategies.

2. Definitions and examples. The probability structure of a controlled (nonMarkov) model is given by a collection $\{X, A(\cdot), p\}$, where $X$ is a countable state space; $A(x)$ is the set of controls admissibile in the state $x \in X$, and is a measurable subset of some set $A$ equipped with a $\sigma$-algebra $\mathscr{A}$ containing all singleton sets in $A$; $p$ is a transition probability. Denote $H_{n}=(X \times A)^{n} \times X, \tilde{H}_{n}=(X \times A)^{n+1}$, where $n=$ $0,1, \cdots$, and $H=\bigcup_{n=0}^{\infty} H_{n}, \tilde{H}=\bigcup_{n=0}^{\infty} \tilde{H}_{n}, H_{\infty}=(X \times A)^{\infty}$. On $H_{\infty}$ consider the $\sigma-$ algebras $\mathscr{F}_{n}, \tilde{\mathscr{F}}_{n}$ and $\mathscr{F}_{\infty}$, generated by the product of the $\sigma$-algebras $2^{X}$ and $\mathscr{A}$. We 
shall also denote the corresponding $\sigma$-algebras on $H, H_{n}$ and $\tilde{H}, \tilde{H}_{n}$ by $\mathscr{F}_{n}$ and $\tilde{F}_{n}$, $n=0,1, \cdots$. On $H$ and $H$ consider the $\sigma$-algebras $\mathscr{F}^{n} \bigvee_{n=0}^{\infty} \mathscr{F}_{n}$ and $\tilde{\mathscr{F}}^{n} \bigvee_{n=0}^{\infty} \tilde{\mathscr{F}}_{n}$, respectively. For each $z \in X$ the function $p\left(z(\cdot): H \rightarrow[0,1]\right.$ is assumed to be $\tilde{F}_{-}$ measurable and $p(X \mid \tilde{h})=1$ for all $\tilde{h} \in \tilde{H}$, where $p(Y \mid \tilde{h})=\sum_{z \in Y} p(z \mid \tilde{h}), Y \subseteq X$.

A strategy $\pi$ is a sequence $\left\{\pi_{n}\right\}_{n=0}^{\infty}$ of transition probabilities from $\left(H_{n}, \mathscr{F}_{n}\right)$ into $(A, \mathscr{A})$ satisfying the condition $\pi_{n}\left(A\left(x_{n}\right) \mid h_{n}\right)=1$ for all $h_{n}=x_{0}, a_{0} \cdots x_{n} \in H_{n}, n=$ $0,1, \cdots$. If for all $h_{n} \in H_{n}, n=0,1, \cdots$, the measure $\pi_{n}\left(\cdot \mid h_{n}\right)$ is concentrated at one point, then the strategy is called nonrandomized. A nonrandomized strategy $\varphi$ is given by a sequence of $\mathscr{F}_{n}$-measurable mappings $\left\{\varphi_{n}\right\}_{n=0}^{\infty}, \varphi_{n}: H_{n} \rightarrow A$.

Let $\mathscr{P}(X)$ be the set of all probability distributions on $X$. For each strategy $\pi \in \Pi$ and each initial distribution $\mu \in \mathscr{P}(X)$ the probability measure $\mathbf{P}_{\mu}^{\pi}$ on $\left(H_{\infty}, \mathscr{F}_{\infty}\right)$ is defined as usual. Let $\mathbf{E}_{\mu}^{\pi}$ denote the expectation calculated with respect to this measure. If $\mu(x)=1$ for some $x \in X$, then we shall write $\mathbf{P}_{x}^{\pi}$ and $\mathbf{E}_{x}^{\pi}$, respectively. We shall also consider the restriction of the measures $\mathbf{P}_{\mu}^{\pi}$ to the measurable spaces $\left(H_{n}, \mathscr{F}_{n}\right)$ and $\left(\tilde{H}_{n}, \tilde{\mathscr{F}}_{n}\right)$.

Note that the case $p(X \mid \tilde{h}) \leqq 1$ also fits into the considered scheme. It suffices to add to $X$ a new absorbing state (for more detail, see for example [21, Remark 2.1]). For this reason, in defining new models (say, embedded models), we shall not require that the condition $p(X \mid \tilde{h})=1$ hold for them.

To study various concrete classes of strategies we shall consider the following construction. Suppose given a countable set $B$ and an $\mathscr{F}$-measurable function $f: H \rightarrow B$. We shall call a strategy $\pi$ a randomized $(f, B)$-generated strategy if for any pair $(x, b) \in X \times B$ there exists a measure $\pi(\cdot \mid x, b)$ on $A$ such that $\pi_{n}\left(\cdot \mid h_{n}\right)=\pi\left(\cdot \mid x_{n}, f\left(h_{n}\right)\right)$ for any prehistory $h_{n} \in H, n=0,1, \cdots$. Denote by $R B^{f}$ the set of all randomized $(f, B)$-generated strategies. If $\pi \in R B^{f}$ and for each pair $(x, b)$ the measure $\pi(\cdot \mid x, b)$ is concentrated at a point, then we shall call such a strategy an $(f, B)$-generated strategy. Let $B^{f}$ denote the set of all $(f, B)$-generated strategies.

Condition 2.1. For any two prehistories $h_{n} \in H_{n}$ and $h_{m} \in H_{m}$ such that $m>n$, $h_{m}=h_{n} a_{n} x_{n+1} \cdots x_{m}$ and $x_{m}=x_{n}$, one has $f\left(h_{n}\right) \neq f\left(h_{m}\right)$.

Let us give examples of $(f, B)$-generated strategies for $f$ satisfying Condition 2.1. For any set $C$ let $1_{C}(\cdot)$ denote the indicator function of $C$. If the argument is $h_{n}, h_{\infty}$ or $\tilde{h}_{n}, n=0,1, \cdots$, and $C$ is given by the condition $\{\cdot\}$, then we shall write $1\{\cdot\}$, instead of $1_{\{\cdot\}}(\cdot)$. For $Y \subseteq X, h_{n} \in H_{n}, n=0,1, \cdots$, let $m\left(h_{n}, Y\right)=\sum_{i=0}^{n} 1\left\{x_{i} \in Y\right\}$ denote the number of hits in $Y$ prior to time $n$ inclusively.

Example 2.1. F-strategies [5]. Suppose given a function $F: X \rightarrow 2^{X}$ such that $x \in F(x)$ for all $x \in X$. Put $B=\{1,2, \cdots\}$ and $f\left(h_{n}\right)=m\left(h_{n}, F\left(x_{n}\right)\right)$. A strategy $\varphi \in B^{f}$ $\left(\pi \in R B^{f}\right)$ is called an $F$-strategy (a randomized $F$-strategy).

Thus, $\varphi_{n}\left(h_{n}\right)=\varphi\left(x_{n}, m\left(h_{n}, F\left(x_{n}\right)\right)\right.$ for $\varphi \in B^{f}$. If $F(x) \equiv X$, then the collection of $F$-strategies coincides with the collection of Markov strategies (i.e., nonrandomized strategies that can be represented as $\left.\varphi_{n}\left(h_{n}\right)=\varphi_{n}\left(x_{n}\right)\right)$. If $F(x)=x$ for all $x \in X$, then the collection of $F$-strategies coincides with the collection of tracking strategies (i.e., nonrandomized strategies that can be represented as $\varphi_{n}\left(h_{n}\right)=\varphi\left(x_{n}, m\left(h_{n},\left\{x_{n}\right\}\right)\right)$. Let $M$ denote the set of all Markov strategies, and $R M$ the set of all randomized Markov strategies.

For $Y \subseteq X, h_{n} \in H_{n}, n=0,1, \cdots$, put

$$
\theta\left(h_{n}, Y\right)= \begin{cases}\max \left\{i: 0 \leqq i \leqq n, x_{n-i} \in Y\right\} & \text { if } m\left(h_{n}, Y\right)>0 \\ n & \text { if } m\left(h_{n}, Y\right)=0\end{cases}
$$

and $\xi\left(h_{n}, Y\right)=n-\theta\left(h_{n}, Y\right) 1\left\{m\left(h_{n}, Y\right)>0\right\}$. Thus, if $m\left(h_{n}, Y\right)>0$, then $\theta\left(h_{n}, Y\right)$ is the time elapsed since the last hit in $Y$ and $\xi\left(h_{n}, Y\right)$ is the time of the last hit in $Y$. 
Example 2.2. $Y$-semi-embedded strategies. Let $Y \subseteq X, B=X \times\{0,1, \cdots,\}^{2}, f\left(h_{n}\right)=$ $\left(x_{\xi\left(h_{n}, Y\right)}, m\left(h_{n}, Y\right), \theta\left(h_{n}, Y\right)\right)$. We call a strategy $\varphi \in B^{f}\left(\pi \in R B^{f}\right) Y$-semi-embedded (randomized $Y$-semi-embedded). Let $\bar{\Pi}^{Y}$ and $R \bar{\Pi}^{Y}$ denote the sets of $Y$-semiembedded strategies and randomized $Y$-semi-embedded strategies, $\bar{\Pi}^{Y} \subseteq R \bar{\Pi}^{Y}$. A strategy $\varphi \in \bar{\Pi}^{Y}$ is given by the equality $\varphi_{n}\left(h_{n}\right)=\varphi\left(x_{n}, x_{\xi\left(h_{n}, Y\right)}, m\left(h_{n}, Y\right), \theta\left(h_{n}, Y\right)\right)$.

Example 2.3. Y-embedded strategies. Let $Y \subseteq X$. Put $B=\{0,1, \cdots\}^{2}, f\left(h_{n}\right)=$ $\left(m\left(h_{n}, Y\right), \theta\left(h_{n}, Y\right)\right)$. We call a strategy $\varphi \in B^{f}\left(\pi \in R B^{f}\right)$ a $Y$-embedded (randomized $Y$-embedded) strategy. Let $\Pi^{Y}$ and $R \Pi^{Y}$ denote the sets of $Y$-embedded and randomized $Y$-embedded strategies, $\Pi^{Y} \subseteq R \Pi^{Y}$. For $\varphi \in \Pi^{Y}$,

$$
\varphi_{n}\left(h_{n}\right)=\varphi\left(x_{n}, m\left(h_{n}, Y\right), \theta\left(h_{n}, Y\right)\right) \text {. }
$$

Thus, for a $Y$-embedded strategy the choice of a control on each step depends on (i) the current state, (ii) the number of hits in $Y$ prior to the current instant, (iii) the time elapsed since the last hit in $Y$. For a $Y$-semi-embedded strategy this choice depends on (i)-(iii) and on (iv) the state at the time of the last hit in $Y$ (if the hit took place). Note that $\Pi^{Y} \subseteq \bar{\Pi}^{Y}, R \Pi^{Y} \subseteq R \bar{\Pi}^{Y}$. Every $X$-embedded or $\{\varnothing\}$-embedded strategy is Markovian. The functions $f$ in Examples 2.1-2.3 satisfy Condition 2.1. Other examples of $(f, B)$-generated strategies satisfying Condition 2.1 are: (a) the class of all strategies in the case of countable $A$ and (b) the class of all strategies for which the choice of the control does not depend on the controls selected earlier [22, Examples 1 and 5].

A nonrandomized strategy $\varphi$ is said to be stationary on $Y, Y \subseteq X$, if $\varphi_{n}\left(h_{n}\right)=$ $\varphi_{m}\left(h_{m}\right)$ for $x_{n}=x_{m} \in Y, h_{n} \in H_{n}, h_{m} \in H_{m}, n, m=0,1, \cdots$. A strategy stationary on $X$ is called stationary. Let $S^{Y}$ denote the set of all strategies stationary on $Y$ and put $S=S^{X}$.

In the nontrivial case when at least one of the sets $A(x)$ consists of more than a point, it is impossible to choose the set $B$ and the function $f$ satisfying Condition 2.1 so that $B^{f}=S$. Indeed, if $B$ and $f$ are chosen so that $B^{f}=S$ (see [22, Example 2]), then $f\left(h_{n}\right)=f\left(h_{m}\right)$ for $x_{n}=x_{m}$, which contradicts Condition 2.1. Let us give yet another example of a class of $(f, B)$ generated strategies where $f$ need not satisfy Condition 2.1.

Example 2.4. $Y$-renewal strategies. Let $Y \subseteq X, B=X \times\{0,1, \cdots\}, f\left(h_{n}\right)=\left(x_{\xi\left(h_{n}, Y\right)}\right.$, $\left.\theta\left(h_{n}, Y\right)\right)$. We call a strategy $\varphi \in B^{f}$ a $Y$-renewal strategy and denote by $R^{Y}$ the class of $Y$-renewal strategies. For $\varphi \in R^{Y}$ we have $\varphi_{n}\left(h_{n}\right)=\varphi\left(x_{n}, x_{\xi\left(h_{n}, Y\right)}, \theta\left(h_{n}, Y\right)\right)$. Thus, for a $Y$-renewal strategy the choice of the control on each step depends on (i) the current state, (ii) the state at the last instant of a hit in $Y$, (iii) the time elapsed since the last hit in $Y$. Moreover, prior to the time of first hit in $Y$ the choice of the control depends only on the current state and the current instant of time. Note that $R^{Y} \subseteq \bar{\Pi}^{Y}$, $R^{Y} \subseteq S^{Y}$ and $R^{X}=S, R^{\{\varnothing\}}=M$.

Remark 2.1. Kertz [28] considered the notion of a renewal plan, which is a broader notion than that of a $Y$-renewal strategy introduced above. More precisely, a nonrandomized strategy $\varphi$ is a $Y$-renewal strategy if and only if, in accordance with Definition 2.1 in [28], $\varphi$ is a renewal plan associated with a strategy $\psi$ and a Markov time $\tau$, where $\tau=\min \left\{n>0: x_{n} \in Y\right\}, \psi$ is a strategy of the form $\psi_{n}\left(h_{n}\right)=\psi\left(x_{0}, x_{n}, n\right)$, and $\psi$ satisfies the condition $\psi(x, y, n)=\psi(z, y, n)$ for all $x, z \in X \backslash Y$.

3. A theorem on decomposition of randomized $(f, B)$-generated strategies. Suppose given the set $B$ and function $f$ introduced above. We shall view the set $B^{f}$ as the set of all functions $\varphi$ on $X \times B$ such that $\varphi(x, b) \in A(x)$ for all $(x, b) \in X \times B$, and $R B^{f}$ as the set of all transition probability measures $\Pi(\cdot \mid x, b)$ from $X \times B$ into $A$ satisfying the condition $\pi(A(x) \mid x, b)=1$ for all $x \in X$ and $b \in B$.

For each $E^{1} \subseteq X \times B$ consider, on the set $B^{f}$, the $\sigma$-algebra $\mathscr{E}_{E^{\prime}}$ generated by the cylindrical sets $\left\{\varphi \in B^{f}: \varphi(x, b) \in A^{\prime}\right\}$, where $(x, b) \in E^{\prime}$ and $A^{\prime} \in \mathscr{A}$; denote the $\sigma$ algebra $\mathscr{E}_{X \times B}$ by $\mathscr{E}$. 
LeMma 3.1. For any probability measure $\mu$ on $X$ and any $C \in \mathscr{F}_{\infty}$, the function $\mathbf{P}_{\mu}^{\varphi}(C) ; B^{f} \rightarrow[0,1]$ is $\mathscr{E}$-measurable.

Proof. Consider the set $B^{f} \times H_{\infty}=B^{f} \times X \times A \times X \times A \cdots$. For each $\varphi \in B^{f}$ the measure $\mathbf{P}_{\mu}^{\varphi}(\cdot)$ can be viewed as a measure on $B^{f} \times H_{\infty}$, generated by the initial state $\varphi$ and the transition probabilities $q_{i}, i=0,1, \cdots$, where $q_{0}(x \mid \varphi)=\mu(x), q_{2 k}(x \mid \varphi$, $\left.\tilde{h}_{k-1}\right)=p\left(x \mid \tilde{h}_{k-1}\right)$ and $q_{2 k-1}\left(A^{\prime} \mid \varphi, h_{k-1}\right)=1,\left(x_{k-1}, f\left(h_{k-1}\right)\right)$ for $x \in X, k=1,2, \cdots, A^{\prime} \in$ $\mathscr{A}$. Noting the theorem of Ionescu Tulcea [23, Prop. V.I.I], to prove the lemma it suffices to prove that the $q_{i}$ are measurable.

The $q_{2 k}, k=0,1, \cdots$, do not depend on $\varphi$ and for $k>0$ are $\tilde{\mathscr{F}}_{k-1}$-measurable, thus also $\left(\mathscr{E} \times \tilde{\mathscr{F}}_{k-1}\right)$-measurable. The functions $q_{2 k-1}\left(A^{\prime} \mid \varphi, h_{k-1}\right)$ are $(\mathscr{E} \times$ $\mathscr{F}_{k-1}$ )-measurable since

$$
1_{A^{\prime}}\left(\varphi\left(x_{k-1}, f\left(h_{k-1}\right)\right)\right)=\sum_{(x, b) \in X \times B} 1_{A^{\prime}}(\varphi(x, b)) 1_{\{(x, b)\}}\left(x_{k-1}, f\left(h_{k-1}\right)\right) .
$$

The lemma is proved.

For $(x, b) \in X \times B$ and $n=0,1, \cdots$ denote $H_{n}^{x, b}=\left\{h_{n} \in H_{n}: x_{n}=x, f\left(h_{n}\right)=b\right\}$.

LeMmA 3.2. Let $f$ and $B$ satisfy Condition 2.1 and suppose given a probability measure $\mu$ on $X$, an index $n=0,1, \cdots$, a pair $(x, b) \in X \times B$ and a set $C \subseteq H_{n}^{x, b}, C \in \mathscr{F}_{n}$. Then the function $\mathbf{P}_{\mu}^{\varphi}(C): B^{f} \rightarrow[0,1]$ is $\mathscr{E}_{X \times B \backslash\{(x, b)\}}$-measurable.

Proof. Consider the set $B^{f} \times H_{n}$. Since $C \subseteq H_{n}^{x, b}$, it follows from Condition 2.1 that the value of $\mathbf{P}_{\mu}^{\varphi}(C)$ is given by the transition probabilities $q_{i}^{\prime}, i=0,1, \cdots, n-1$, where $q_{2 k}^{\prime}=q_{2 k}$, and

$$
\begin{aligned}
q_{2 k-1}^{\prime}\left(A^{\prime} \mid \varphi, h_{k-1}\right) & =q_{2 k-1}\left(A^{\prime} \mid \varphi, h_{k-1}\right) 1_{X \times B \backslash\{(x, b)\}}\left(x_{k-1}, f\left(h_{k-1}\right)\right) \\
& =\sum_{\left(x^{\prime}, b^{\prime}\right) \in X \times B \backslash\{(x, b)\}} 1_{A^{\prime}}\left(\varphi\left(x^{\prime}, b^{\prime}\right)\right) 1_{\left\{\left(x^{\prime}, b^{\prime}\right)\right\}}\left(x_{k-1}, f\left(h_{k-1}\right)\right) .
\end{aligned}
$$

The transition measures $q_{2 k}^{\prime}$ do not depend on $\varphi$ and are $\tilde{\mathscr{F}}_{k-1}$-measurable (see the proof of Lemma 3.1). It follows from (3.1) that the transition measures $q_{2 k-1}^{\prime}$ are $\left(\mathscr{E}_{X \times B \backslash\{(x, b)\}} \times \mathscr{F}_{k-1}\right)$-measurable. The measurability of the transition measures $q_{i}^{\prime}$ implies Lemma 3.2. The lemma is proved.

Let $\pi \in R B^{f}$. According to the theorem on the product of probabilities [23, Prop. VI.2], there exists on $\left(B^{f}, \mathscr{E}\right)$ a unique measure $m^{\pi}$ generated by the product of the measures $\pi(\cdot \mid x, b)$ over the set of all pairs $(x, b) \in X \times B$. Obviously if $E^{\prime} \cap E^{\prime \prime}=\varnothing$, where $E^{\prime}, E^{\prime \prime} \subset X+B$, then the $\sigma$-algebras $\mathscr{E}_{E^{\prime}}$ and $\mathscr{E}_{E^{\prime \prime}}$ are independent with respect to the measure $m^{\pi}$. From the definition of $m^{\pi}$ it follows that for any $A^{\prime} \in \mathscr{A}, \pi \in R B^{f}$ and $(x, b) \in X \times B$, we have

$$
\pi\left(A^{\prime} \mid x, b\right)=m^{\pi}\left\{\left(\varphi: \varphi(x, b) \in A^{\prime}\right\}=\int_{B^{f}} 1_{A^{\prime}}(\varphi(x, b)) m^{\pi}(d \varphi) .\right.
$$

THeOREM 3.1. If $f$ and $B$ satisfy Condition 2.1 , then, for any $\mu \in \mathscr{P}(X), C \in \mathscr{F}_{\infty}$ and $\pi \in R B^{f}$

$$
\mathbf{P}_{\mu}^{\pi}(C)=\int_{B^{f}} \mathbf{P}_{\mu}^{\varphi}(C) m^{\pi}(d \varphi)
$$

Proof. We shall follow the proof of the similar assertions in [1], [20]. By the theorem on continuation of a measure, it suffices to prove that if for any $n=0,1, \cdots$ we consider the restriction of the measures $\mathbf{P}_{\mu}$ to $\mathscr{F}_{n}$, then (3.3) is valid for each $C \in \mathscr{F}_{n}$. For $n=0$, (3.3) follows for any $C \subseteq H_{0}=X$ from the fact that $\mathbf{P}_{\mu}^{\sigma}(C)=\mu(C)$ for any strategy $\sigma \in \Pi$. 
Suppose that for some $n=0,1, \cdots$ equality (3.3) has been proved for all $C \in \mathscr{F}_{n}$. Let $A^{\prime} \in \mathscr{A}$. Then, using (3.2) and the inductive hypothesis we have

$$
\begin{aligned}
\mathbf{P}_{\mu}^{\pi}\left(C \times A^{\prime}\right) & =\int_{C} \pi_{n}\left(A^{\prime} \mid h_{n}\right) \mathbf{P}_{\mu}^{\pi}\left(d h_{n}\right)=\sum_{(x, b) \in X \times B} \pi\left(A^{\prime} \mid x, b\right) \mathbf{P}_{\mu}^{\pi}\left(H_{n}^{x, b} \cap C\right) \\
& =\sum_{(x, b) \in X \times B} \int_{B^{f}} 1_{A^{\prime}}(\varphi(x, b)) m^{\pi}(d \varphi) \int_{B^{f}} \mathbf{P}_{\mu}^{\varphi}\left(H_{n}^{x, b} \cap C\right) m^{\pi}(d \varphi) \\
& =\int_{B^{f}(x, b) \in X \times B} \sum_{A^{\prime}}(\varphi(x, b)) \mathbf{P}_{\mu}^{\varphi}\left(H_{n}^{x, b} \cap C\right) m^{\pi}(d \varphi) .
\end{aligned}
$$

(The last equality follows from the fact that the function $1_{A^{\prime}}(\varphi(x, b))$ is $\mathscr{E}_{\{(x, b)\}^{-}}$ measurable with respect to $\varphi$, the function $\mathbf{P}_{\mu}^{\varphi}\left(H_{n}^{x, b} \cap C\right)$ is $\mathscr{E}_{X \times B \backslash\{(x, b)\}}$-measurable by Lemma 3.2, and the $\sigma$-algebras $\mathscr{E}_{\{(x, b)\}}$ and $\mathscr{E}_{X \times B \backslash\{(x, b)\}}$ are independent with respect to the measure $m^{\pi}$. In the derived expression, the integration and summation can be interchanged.)

For any $\varphi \in B^{f}$ we have

$$
\begin{aligned}
\mathbf{P}_{\mu}^{\varphi}\left(C \times A^{\prime}\right) & =\int_{C} 1_{A^{\prime}}\left(\varphi_{n}\left(h_{n}\right)\right) \mathbf{P}_{\mu}^{\varphi}\left(d h_{n}\right) \\
& =\sum_{(x, b) \in X \times B} 1_{A^{\prime}}(\varphi(x, b)) \mathbf{P}_{\mu}^{\varphi}\left(H_{n}^{x, b} \cap C\right) .
\end{aligned}
$$

Substituting (3.5) into the last of equalities (3.4), we find that

$$
\mathbf{P}_{\mu}^{\pi}\left(C \times A^{\prime}\right)=\int_{B^{f}} P_{\mu}^{\varphi}\left(C \times A^{\prime}\right) m^{\pi}(d \varphi)
$$

for all $C \in \mathscr{F}_{n}$ and $A^{\prime} \in \mathscr{A}$. Hence,

$$
\mathbf{P}_{\mu}^{\pi}(\tilde{C})=\int_{B^{f}} \mathbf{P}_{\mu}^{\varphi}(\tilde{C}) m^{\pi}(d \varphi), \quad \tilde{C} \in \mathscr{F}_{n} .
$$

Let $\tilde{C} \in F_{n}, X^{\prime} \subseteq X$. Then, using Fubini's theorem in the form of [24, Chap. II, Thm. 16] we have

$$
\begin{aligned}
\mathbf{P}_{\mu}^{\pi}\left(\tilde{C} \times X^{\prime}\right) & =\int_{\tilde{C}} p\left(X^{\prime} \mid \tilde{h_{n}}\right) \mathbf{P}_{\mu}^{\pi}\left(d \tilde{h}_{n}\right) \\
& =\int_{B^{f}} m^{\pi}(d \varphi) \int_{\tilde{C}} p\left(X^{\prime} \mid \tilde{h}_{n}\right) \mathbf{P}_{\mu}^{\varphi}\left(d \tilde{h}_{n}\right) \\
& =\int_{B^{f}} \mathbf{P}_{\mu}^{\varphi}\left(\tilde{C} \times X^{\prime}\right) m^{\pi}(d \varphi) .
\end{aligned}
$$

Since $\tilde{C} \in \mathscr{F}_{n}$ and $X^{\prime} \subseteq X$ are arbitrary, (3.3) is valid for all $C \in \mathscr{F}_{n+1}$. The theorem is proved.

Suppose given a measurable function $R: H_{\infty} \rightarrow[-\infty,+\infty]$. Consider the quantity $\mathbf{E}_{\mu}^{\pi} R$, where if $R$ is a nonintegrable function (i.e., both integrals of the positive and negative parts of $R$ are infinite), then $\mathbf{E}_{\mu}^{\pi} R=-\infty$. For an arbitrary number $c$ put $c^{+}=\max \{0, c\}, c^{-}=\min \{0, c\}$. 
If $\mathbf{E}_{\mu}^{\pi} R^{-}>-\infty$, then from Theorem 3.1 and the lemma on multiplicative systems [4, Addendum 5], [24, Chap. I, Thm. 20] applied to $R^{+}$and $R^{-}$, we have

$$
\mathbf{E}_{\mu}^{\pi} R=\int_{B^{f}}\left(\mathbf{E}_{\mu}^{\varphi} R\right) m^{\pi}(d \varphi) .
$$

This gives us the following assertion.

COROllary 3.1. If $f$ and $B$ satisfy Condition 2.1, then for any $\pi \in R B^{f}, \mu \in \mathscr{P}(X)$ and $N \in\left[-\infty,+\infty\left[\right.\right.$ there exists a strategy $\varphi \in B^{f}$ such that

$$
\boldsymbol{E}_{\mu}^{\varphi} R \geqq\left\{\begin{array}{cl}
\mathbf{E}_{\mu}^{\pi} R & \text { if } \mathbf{E}_{\mu}^{\pi} R<+\infty, \\
N & \text { if } \mathbf{E}_{\mu}^{\pi} R=+\infty
\end{array}\right.
$$

Remark 3.1. For the class of stationary strategies the decomposition theorem is not true. Indeed, let $X=\{x\}, A(x)=\{0,1\}, p(x \mid \tilde{h})=1$, where $\tilde{h} \in \tilde{H}$. Consider a randomized stationary strategy $\pi$ for which each of the controls 0 and 1 on each step is chosen with probability $\frac{1}{2}$. The validity of the decomposition of $\pi$ with respect to stationary strategies would mean that the measure $\mathbf{P}_{x}^{\pi}$ is concentrated on two trajectories $x, 0, x, 0, \cdots$ and $x, 1, x, 1, \cdots$, which is not true.

Remark 3.2. A description of all classes of strategies for which the decomposition theorem is true remains an open question. For example, under the assumptions of this paper the possibility of decomposing an arbitrary strategy in $\Pi$ remains unproven. In this case the set $B^{f}=H$ on the basis of whose elements the controls are chosen is no longer countable, and additional difficulties arise that are connected with measurability. For models with Borel state and control spaces, such a theorem has been proved in [19] (see also [20]). However, above it was not assumed that $\boldsymbol{A}$ is a Borel space. At the same time, in studying Markov models with total criterion one can confine oneself to Theorem 3.1, since in this case for any strategy there exists a randomized $(f, B)$ generated strategy of a special form (a randomized $Y$-embedded strategy (Theorem 4.1), in particular, a randomized Markov strategy [18]) with the same payoff as the original strategy.

4. The sufficiency of $\boldsymbol{Y}$-embedded strategies in Markov models. Throughout what follows we consider a homogeneous Markov model $\mathcal{M}=\{X, A(\cdot), p, r\}$, where $X, A(\cdot)$, $p$ are defined in $\S 2$, and $p\left(\cdot \mid \tilde{h}_{n}\right)=p\left(\cdot \mid x_{n}, a_{n}\right)$ for all $\tilde{h}^{n} \in \tilde{H}_{n}, n=0,1, \cdots, r(x, a)$ is the reward function, $-\infty \leqq r(x, a) \leqq+\infty$ for all $x \in X, a \in A(x)$. It is assumed that $r(x, a)$ is measurable with respect to $a$ (the measurability of $p(\cdot \mid x, a)$ in $a$ follows from the definition in $\S 2$ ).

For any $\pi \in \Pi$ and $\mu \in \mathscr{P}(X)$ we define

$$
\begin{gathered}
w_{+}^{\pi}(\mu)=\mathbf{E}_{\mu}^{\pi} \sum_{n=0}^{\infty} r^{+}\left(x_{n}, a_{n}\right), \\
w_{-}^{\pi}(\mu)=\mathbf{E}_{\mu}^{\pi} \sum_{n=0}^{\infty} r^{-}\left(x_{n}, a_{n}\right), \\
w^{\pi}(\mu)=\mathbf{E}_{\mu}^{\pi} \sum_{n=0}^{\infty} r\left(x_{n}, a_{n}\right)=w_{+}^{\pi}(\mu)+w_{-}^{\pi}(\mu),
\end{gathered}
$$

where $w^{\pi}(\mu)=-\infty$, if the right-hand side of (4.3) is indeterminate of the form $(+\infty)+(-\infty)$. Denote $v(\mu)=\sup _{x \in \Pi} w^{\pi}(\mu)$. If $\mu$ is concentrated at $x \in X$, we shall write $w^{\pi}(x)$ and $v(x)$ instead of $w^{\pi}(\mu)$ and $v(\mu)$.

Fix $Y \subseteq X, Y \neq \varnothing$. Consider the sequence of Markov times $\tau^{i}: \tau^{0}\left(h_{\infty}\right)=0$, $\tau^{i+1}\left(h_{\infty}\right)=\inf \left\{n>\tau^{i}\left(h_{\infty}\right) ; x_{n} \in Y\right\}$, where $h_{\infty} \in H, i=0,1, \cdots$. 
THEOREM 4.1. For $\mu \in \mathscr{P}(X)$ and $\pi \in \Pi$ consider a randomized $Y$-embedded strategy $\sigma$ such that, for all $A^{\prime} \in \mathscr{A}, x \in X, m, k=0,1, \cdots$,

$$
\sigma\left(A^{\prime} \mid x, m, k\right)=\mathbf{P}_{\mu}^{\pi}\left\{a_{\tau} m_{+k} \in A^{\prime} \mid x_{\tau} m_{+k}=x, \tau^{m+1}>\tau^{m}+k\right\}\left(\mathbf{P}_{\mu}^{\pi} \text {-a.s. }\right) .
$$

Then $w^{\sigma}(\mu)=w^{\pi}(\mu)$ and, for all $A^{\prime} \in \mathscr{A}, x \in X, m, k=0,1, \cdots$,

$$
\begin{aligned}
\mathbf{P}_{\mu}^{\sigma}\left\{x_{\tau} m_{+k}\right. & \left.=x, \tau^{m+1}>\tau^{m}+k, a_{\tau} m_{+k} \in A^{\prime}\right\} \\
& =\mathbf{P}_{\mu}^{\pi}\left\{x_{\tau} m_{+k}=x, \tau^{m+1}>\tau^{m}+k, a_{\tau} m_{+k} \in A^{\prime}\right\} .
\end{aligned}
$$

For $Y=X$, Theorem 4.1 is the following familiar result.

CoRollary 4.1 [18]. Suppose given for some $\pi \in \Pi$ and $\mu \in \mathscr{P}(X)$ a Markov strategy $\sigma$ such that, for all $A^{\prime} \in \mathscr{A}, x \in X, m=0,1, \cdots$,

$$
\sigma\left(A^{\prime} \mid x, m\right)=\mathbf{P}_{\mu}^{\pi}\left\{a_{m} \in A^{\prime} \mid x_{m}=x\right\}\left(\mathbf{P}_{\mu}^{\pi} \text {-a.s. }\right) .
$$

Then $w^{\sigma}(\mu)=w^{\pi}(\mu)$ and, for all $A^{\prime} \in \mathscr{A}, x \in x, m=0,1, \cdots$,

$$
\mathbf{P}_{\mu}^{\sigma}\left\{x_{m}=x, a_{m} \in A^{\prime}\right\}=\mathbf{P}_{\mu}^{\pi}\left\{x_{m}=x, a_{m} \in A^{\prime}\right\}
$$

Proof of Theorem 4.1. For any randomized $Y$-embedded strategy $\sigma$,

$$
\begin{aligned}
\mathbf{P}_{\mu}^{\sigma}\left\{x_{\tau} m_{+k}\right. & \left.=x, \tau^{m+1}>\tau^{m}+k, a_{\tau} m_{+k} \in A^{\prime}\right\} \\
& =\mathbf{P}_{\mu}^{\sigma}\left\{x_{\tau} m_{+k}=x, \tau^{m+1}>\tau^{m}+k\right\} \sigma\left(A^{\prime} \mid x, m, k\right) .
\end{aligned}
$$

This and (4.4) imply that, for any $x, m$ and $k$, equality (4.5) for all $A^{\prime} \in \mathscr{A}$ is equivalent to the equality

$$
\mathbf{P}_{\mu}^{\sigma}\left\{x_{\tau} m_{+k}=x, \tau^{m+1}>\tau^{m}+k\right\}=\boldsymbol{P}_{\mu}^{\pi}\left\{x_{\tau} m_{+k}=x, \tau^{m+1}>\tau^{m}+k\right\} .
$$

The proof of (4.6) is carried out by induction on $m$ and $k$ using, for an arbitrary strategy $\tilde{\pi} \in \Pi$, the equality

$$
\begin{aligned}
& \mathbf{P}_{\mu}^{\tilde{\pi}}\left\{x_{\tau} m_{+k+1}=x, \tau^{m+1}>\tau^{m}+k+1\right\} \\
& \quad=\sum_{z \in X} \int_{A} p\left(x \mid z, a_{\tau} m_{+k}\right) \mathbf{P}_{\mu}^{\tilde{\pi}}\left\{x_{\tau} m_{+k}=z, \tau^{m+1}>\tau^{m}+k, d a_{\tau} m_{+k}\right\}
\end{aligned}
$$

in passing from $(m, k)$ to $(m, k+1)$ and the equality

$$
\mathbf{P}_{\mu}^{\tilde{\pi}}\left\{x_{\tau^{m+1}}=x\right\}=\sum_{z \in X} \sum_{k=0}^{\infty} \int_{A} p\left(x \mid z, a_{\tau} m_{+k}\right) \mathbf{P}_{\mu}^{\tilde{\pi}}\left\{x_{\tau} m_{+k}=z, \tau^{m+1}>\tau^{m}+k, d a_{\tau} m_{+k}\right\}
$$

in passing from $m$ to $m+1$.

Let us show that the equality $w^{\sigma}(\mu)=w^{\pi}(\mu)$ follows from (4.5). Note that in the series on the right-hand side of (4.1) and (4.2), one can arbitrarily permute or group terms. Hence for any strategy $\tilde{\pi} \in \Pi$ we have

$$
\begin{aligned}
w_{+}^{\tilde{\pi}}(\mu) & =\mathbf{E}_{\mu}^{\tilde{\pi}} \sum_{m=0}^{\infty} \sum_{n=\tau^{m}}^{\tau^{m+1}-1} r^{+}\left(x_{n}, a_{n}\right) \\
& =\sum_{m=0}^{\infty} \sum_{k=0}^{\infty} \mathbf{E}_{\mu}^{\tilde{\pi}} 1\left\{\tau^{m+1}>\tau^{m}+k\right\} r^{+}\left(x_{\tau^{m}+k}, a_{\tau^{m}+k}\right) .
\end{aligned}
$$

But by (4.5), the distributions of the random variable $1\left\{\tau^{m+1}>\tau^{m}+k\right\} r^{+}\left(x_{\tau^{m}+k}, a_{\tau^{m}+k}\right)$ with respect to the measures $\mathbf{P}_{\mu}^{\sigma}$ and $\mathbf{P}_{\mu}^{\pi}$ coincide for each $m$ and $k$. Consequently, $w_{+}^{\sigma}(\mu)=w_{+}^{\pi}(\mu)$. Similarly, $w_{-}^{\sigma}(\mu)=w_{-}^{\pi}(\mu)$. The theorem is proved. 
Remark 4.1. Krylov [25, Thm. 1] derived a profound result that can be interpreted as follows: in controlled diffusion models with infinite horizon for fixed initial state, there exists for an arbitrary strategy a randomized stationary strategy with the same expected total payoff. Apparently, Theorem 4.1 can be proved as a corollary of the discrete-time analogue of this result using the construction of the evolvent described in $[22, \S 4]$.

Applying Corollary 3.4 to the function

$$
R\left(h_{\infty}\right)= \begin{cases}\sum_{n=0}^{\infty} r\left(x_{n}, a_{n}\right) & \text { for } \sum_{n=0}^{\infty} r^{-}\left(x_{n}, a_{n}\right)>-\infty, \\ -\infty & \text { for } \sum_{n=0}^{\infty} r^{-}\left(x_{n}, a_{n}\right)=-\infty,\end{cases}
$$

we obtain the following assertion.

Corollary 4.2. For any $\mu \in \mathscr{P}(X), \pi \in \Pi, N \in[-\infty,+\infty[$ and $Y \subseteq X$ there is a $Y$-embedded strategy $\varphi$ such that

$$
w^{\varphi}(\mu) \geqq \begin{cases}w^{\pi}(\mu) & \text { if } w^{\pi}(\mu)<+\infty, \\ N & \text { if } w^{\pi}(\mu)=+\infty,\end{cases}
$$

(in the particular case $Y=X$ the strategy $\varphi$ is Markovian).

Corollary 4.3. For all $\mu \in \mathscr{P}(X)$ and $Y \subseteq X$,

$$
v(\mu)=\sup _{\varphi \in \Pi_{Y}^{Y}} w^{\varphi}(\mu)=\sup _{\varphi \in \Pi_{Y}^{Y}} w^{\varphi}(\mu)=\sup _{\varphi \in M} w^{\varphi}(\mu) .
$$

(The second equality in the corollary follows from the inclusions $\Pi^{Y} \subseteq \bar{\Pi}^{Y} \subseteq \Pi$, and the last one from the first if we set $Y=\varnothing$.)

Remark 4.2. The equality $v(x)=\sup _{\varphi \in M} w^{\varphi}(x)$ is well known [26], [20], [27], although it does not formally follow from these articles. Indeed, it was assumed in [26] that

$$
w_{+}^{\pi}(x)<+\infty \text { for all } x \in X, \pi \in \Pi,
$$

while [20] and [27] considered a model with Borel state and control spaces. However, in our article the space $A$ need not be Borelian.

For some $B$ and $f$ satisfying Condition 2.1, consider the sets $B^{f}$ and $R B^{f}$. For $(x, b) \in X \times B$ define the Markov time $\tau^{x, b}\left(h_{\infty}\right)=\min \left\{n \geqq 0: x_{n}=x, f\left(h_{n}\right)=b\right\}$. In case $B^{f}=\Pi^{Y}$ and $R B^{f}=R \Pi^{Y}$ (i.e., in the situation of Example 2.3) equalities (4.4) and (4.5) can be rewritten as follows:

$$
\begin{gathered}
\sigma(A \mid x, b)=\mathbf{P}_{\mu}^{\pi}\left\{a_{\tau^{x, b}} \in A^{\prime} \mid \tau^{x, b}<\infty\right\}\left(\mathbf{P}_{\mu}^{\pi} \text {-a.s. }\right), \\
\mathbf{P}_{\mu}^{\sigma}\left\{\tau^{x, b}<\infty, a_{\tau^{x, b}} \in A^{\prime}\right\}=\mathbf{P}_{\mu}^{\pi}\left\{\tau^{x, b}<\infty, a_{\tau^{x, b}} \in A^{\prime}\right\}\left(\mathbf{P}_{\mu}^{\pi} \text {-a.s. }\right) .
\end{gathered}
$$

The question arises as to whether the assertion of Theorem 4.1 remains true in the case of arbitrary $(f, B)$-generated strategies when (4.4) is replaced by (4.8) and (4.5) by (4.9). The following example pertaining to tracking strategies (i.e., $f\left(h_{n}\right)=$ $\left.m\left(h_{n},\left\{x_{n}\right\}\right)\right)$ gives a negative answer to this question.

Example 4.1. Let $X=\{0,1\}, \quad A=A(i)=\left\{a^{0}, a^{1}\right\}, \quad p\left(i \mid j, a^{i}\right)=1$, where $i, j=$ $0,1, r\left(0, a^{1}\right)=-1$ and $r\left(0, a^{0}\right)=r\left(1, a^{i}\right)=0, i=0,1$. Let $\mu(0)=\mu(1)=\frac{1}{2}$ and $\pi$ be a Markov strategy for which on the step 0 the controls $a^{0}$ and $a^{1}$ are chosen with probability $\frac{1}{2}$ in both states, while on the steps $1,2, \cdots$ the control $a^{i}$ is chosen with probability 1 in the state $x^{i}$. It is easy to check that $w^{\pi}(\mu)=-\frac{1}{4}$, while, for the corresponding tracking strategy $\sigma$ defined by $(4.9), w^{\sigma}(\mu)=-\frac{2}{9}$. 
5. Embedded models. Throughout below we assume that (4.7) holds.

Note that an arbitrary Markov strategy $\varphi \in M$ can be described as a mapping $\varphi: X \times\{0,1, \cdots\} \rightarrow A$ such that $\varphi(x, n) \in A(x)$, and as a sequence of mappings $\varphi=$ $\left\{\varphi_{0}, \varphi_{1}, \cdots\right\}, \varphi_{n}(x) \in A(x)$. Both descriptions are equivalent and we shall use whichever one of them suits us in a particular situation. If we use the first description, then $M=B^{f}$ (see Example 2.1 or [22, Example 3]) and a $\sigma$-algebra $\mathscr{E}$ is given on $M$. Fix $Y \subseteq X, Y \neq \varnothing$. For $\varphi \in M$ and $x, z \in Y$ define the quantities

$$
\begin{aligned}
p_{Y}(z \mid x, \varphi) & =\mathbf{P}_{x}^{\varphi}\left\{\tau<\infty, x_{\tau}=z\right\}, \\
r_{Y}(x, \varphi) & =\mathbf{E}_{x}^{\varphi} \sum_{n=0}^{\tau-1} r\left(x_{n}, a_{n}\right),
\end{aligned}
$$

where $\tau=\min \left\{n>0: z_{n} \in Y\right\}$. Due to Lemma 3.1, $p_{Y}$ and $r_{Y}$ are measurable with respect to $\varphi$.

We call the controlled Markov model $M_{Y}=\left\{Y, A_{Y}(\cdot), p_{Y}, r_{Y}\right\}$, where $A_{Y}(x)=M$ for all $x \in Y$, a $Y$-embedded model. For this model we shall write $M_{Y}, S_{Y}, w_{Y}(\cdot)$, and $v_{Y}(\cdot)$ instead of $M, S, w^{\cdot}(\cdot)$ and $v(\cdot)$, respectively. Let $s(x)=\sup \left\{w^{\varphi}(x): \varphi \in S\right\}$ and $s_{Y}(\cdot)$ be the pure value of the strategies in the model $M_{Y}$.

THEOREM 5.1. For all $Y \subseteq X$ and $y \in Y$, the following equalities are valid:

(A) $v(y)=v_{Y}(y)$

(B) $\sup \left\{w^{\varphi}(y): \varphi \in R^{Y}\right\}=s_{Y}(y)$.

Proof. A strategy $\varphi \in \bar{\Pi}^{Y}$ is uniquely given by the function $\varphi(z, x, m, k)$ mapping $X^{2} \times\{0,1, \cdots\}^{2}$ into $A$. A strategy $\psi \in M_{Y}$ is uniquely given by the collection $\left\{\psi_{m}(z)\right\}$, where $z \in Y, m=0,1, \cdots$, and $\psi_{m}(z) \in M$. Denote $\psi^{m, z}=\psi_{m}(z)$, where $\psi^{m, z}: X \times$ $\{0,1, \cdots\} \rightarrow A$. Putting $\psi^{m-1, z}(x, k)=\varphi(z, x, m, k)$, we obtain a mapping of $\bar{\Pi}^{Y}$ onto $M_{Y}$. Here the set $R^{Y}$ is mapped onto $S_{Y}$. For strategies $\varphi \in \bar{\Pi}^{Y}$ and $\psi \in M_{Y}$, we have

$$
\begin{aligned}
w^{\varphi}(y) & =\mathbf{E}_{y}^{\varphi} \sum_{n=0}^{\infty} r\left(x_{n}, a_{n}\right)=\mathbf{E}_{y}^{\varphi} \sum_{m=1}^{\infty} \sum_{n=\tau^{m-1}}^{\tau^{m}-1} r\left(x_{n}, a_{n}\right) \\
& =\mathbf{E}_{y}^{\psi} \sum_{m=0}^{\infty} r_{Y}\left(x_{m}, \psi_{m}\right)=w_{Y}^{\psi}(y)
\end{aligned}
$$

for $y \in Y$, where $\tau^{0}\left(h_{\infty}\right)=0, \tau^{m+1}\left(h_{\infty}\right)=\min \left\{n>\tau^{m}\left(h_{\infty}\right), x_{n} \in Y\right\}$. This and Corollary 4.3 applied to the class of strategies $\bar{\Pi}^{Y}$ in the model $M$ and to the class $M_{Y}$ in the model $M_{Y}$ give us Assertion (A) of the theorem. Since under the established correspondence $R^{Y}$ is mapped onto $S_{Y}$, Assertion (B) follows from (5.1).

The author expresses his profound gratitude to E. I. Presman and I. M. Sonin for a number of useful remarks.

\section{REFERENCES}

[1] N. V. KRYLOV, The construction of an optimal strategy for a finite controlled chain, Theory Probab. Appl., 10 (1965), pp. 45-54.

[2] D. BlaCkwell, Discrete dynamic programming, Ann. Math. Statist., 33 (1962), pp. 719-726.

[3] - Discrete dynamic programming, Ann. Math. Statist., 36 (1965), pp. 226-235.

[4] E. B. Dynkin ANd A. A. Yushkevich, Controlled Markov Processes, Springer, New York, 1979.

[5] I. M. SONIN AND E. A. FAINBERG, Sufficient classes of strategies in countable controlled Markov chains with total criterion, Dokl. Akad. Nauk SSSR, 275 (1984), pp. 806-809. (In Russian.)

[6] T. P. Hill, On the existence of good Markov strategies, Trans. Amer. Math. Soc., 247 (1979), pp. 157-176.

[7] H. Everetr, Recursive games, Ann. of Math. Stud., 39 (1957), pp. 47-78.

[8] R. YA. Chitashvili, On the existence of $\varepsilon$-optimal stationary policies for a controlled Markov chain, Soobschch. Akad. Nauk Gruzin SSR, 83 (1976), pp. 549-552. (In Russian.) 
[9] A. A. Yushevich AND R. YA. Chitashvili, Controlled random sequences and Markov chains, Uspekhi Mat. Nauk, 37 (1982), pp. 213-242. (In Russian.)

[10] D. ORnsteIn, On the existence of stationary optimal strategies, Proc. Amer. Math. Soc., 20 (1969), pp. 563-569.

[11] S. Demko AND T. P. Hill, Decision processes with total-cost criteria, Ann. Probab., 9 (1981), pp. $293-301$.

[12] J. VAN DER WAL, On stationary strategies in countable state total reward Markov decision processes, Math. Oper. Res., 9 (1984), pp. 290-300.

[13] R. E. StrauCH, Negative dynamic programming, Ann. Math. Statist., 37 (1966), pp. 871-890.

[14] J. VAN DER WAL, On uniformly nearly-optimal Markov strategies, Operations Research Proceedings, 1982, Springer-Verlag, Berlin, 1983, pp. 461-467.

[15] E. A. FAINBERG AND I. M. Sonin, Stationary and Markov policies in countable state dynamic programming, Lecture Notes in Math., 1021 (1983), pp. 111-129.

[16] I. M. SONIN, The existence of a uniformly nearly-optimal Markov strategy for a controlled Markov chain with countable state space, in Models and Methods of Stochastic Optimization, TsEMI AN SSSR, 1984, pp. 213-232. (In Russian).

[17] J. VAN DER WAL AND J. WESSEls, On the use of information in Markov decision processes, Statist. Decisions, 1-2 (1984), pp. 1-21.

[18] C. Derman AND R. E. STRAuCh, A note on memoryless rules for controlling sequential processes, Ann. Math. Statist., 37 (1966), pp. 276-278.

[19] I. I. GikhMAN AND A. V. Skorokhod, Controlled Random Processes, Springer-Verlag, New York, et al., 1979.

[20] E. A. FAINBERG, Nonrandomized Markov and semi-Markov strategies in dynamic programming, Theory Probab. Appl., 27 (1982), pp. 116-126.

[21] J. VAN NUNEN AND J. WESSELS, Markov decision processes with unbounded rewards (Markov Decision Theory), Math. Centre Tracts, 93 (1976), pp. 1-24.

[22] E. A. FAInberg AND I. M. Sonin, Persistently nearly optimal strategies in stochastic dynamic programming, in Statistics and Control of Stochastic Processes (Steklov Seminar 1984), Optimization Software, New York, 1985, pp. 69-101.

[23] J. NeVeu, Mathematical Foundations of the Calculus of Probabilities, Holden-Day, San Francisco, 1965.

[24] P. A. MeYer, Probability and Potentials, Blaisdell, Waltham, MA, 1966.

[25] N. V. KRYLOV, Once more about the connection between elliptic operators and Itô's stochastic equations, in Statistics and Control of Stochastic Processes (Steklov Seminar 1984), Optimization Software, New York, 1985, pp. 214-229.

[26] K. M. VAN HEE, Markov strategies in dynamic programming, Math. Oper. Res., 3 (1978), pp. 37-41.

[27] E. A. FAINBERG, Controlled Markov processes with arbitrary numerical criteria, Theory. Probab. Appl., 27 (1982), 486-503.

[28] R. P. KERTZ, Renewal plans and persistent optimality in countably additive gambling, Math. Oper. Res., 7 (1982), pp. 361-382. 